\title{
Estudio experimental de microaglomerado asfáltico antiderrapante modificado con NFU
}

Experimental study of non-skid microsurfacing asphalt modified with recycled tires

Fecha de entrega: 27 de mayo 2012 Fecha de aceptación: 26 de agosto 2013

\section{Gerardo Botasso ${ }^{1}$ y Adrián Segura ${ }^{2}$}

${ }^{1}$ LEMaC Centro de Investigación Vial, Universidad Tecnológica Nacional, Facultad Regional La Plata, Calle 60 y 124 (1900), La Plata, Prov. de Buenos Aires, Argentina, gbotasso@frlp.utn.edu.ar

${ }^{2}$ Universidad Tecnológica Nacional, Facultad Regional Córdoba, Maestro M. López esq. Cruz Roja Argentina Ciudad Universitaria. Casilla de Correo 36 C.P. (X5016ZAA) Córdoba, Argentina, adrian-segura@hotmail.com

Dadas las actuales mayores velocidades de circulación en vias urbanas y rurales, tipologías de vehiculos y aumento de accidentes por excesos de velocidad, son necesarios nuevos diseños de mezclas asfálticas que permitan mayor adherencia neumático calzada y mejor evacuación del agua de la calzada para asi disminuir las distancias de frenado. Para tal fin se ha considerado utilizar micromezclas en caliente en donde, a efectos de garantizar una adecuada macro y microtextura, se otorgan discontinuidades en la curva granulométrica y en la relación tamaño máximo de árido versus espesor de la capa especial. Sin duda para que estas condiciones de regularidad superficial permanezcan en el tiempo y no se deterioren bajo la acción de las cargas del tránsito y de las condiciones ambientales, es necesario utilizar un ligante asfáltico modificado con polimero. En el presente trabajo se presenta la factibilidad de modificar el asfalto con polvo proveniente del reciclado de Neumáticos Fuera de Uso NFU. Se estudia experimentalmente el desempeño de este aglomerado en comparación a otro considerado como blanco comparativo en donde el ligante asfáltico se modifica con un polímero virgen convencional, como lo es el estireno butadieno estireno, conocido como $S B S$, de mucho mayor costo sin considerar los beneficios del proceso de reciclado del caucho de los neumáticos. Se concluye que los cambios producidos fundamentalmente en la microtextura es uno de los puntos más favorables en la disminución de la distancia de frenado.

Palabras clave: neumáticos fuera de uso, reciclado, antiderrapante, fricción, microaglomerado
Given the current high traffic speeds in urban and rural roads, typologies of vehicles and the elevated number of accidents caused by the excess of speed, it is necessary to create new designs of asphalt mixtures that allow a greater adherence between tire and roadway and evacuate quickly the water from the roadway, hence a braking distance reduction. With that scope it has been considered the use of hot micro mixtures that guarantee an adequate macro and micro texture, by defining discontinuities in the grain size distribution curve and a relationship between the arid maximum size versus layer thickness. Of course, in order to maintain these conditions of superficial regularity along the time without being deteriorated under the action of the traffic load and environmental conditions, it is necessary to use an asphalt binder modified with a polymer. This paper presents the feasibility of modifying the asphalt with dust obtained from the recycling of tires out of use. The performance of this agglomerate compared to another selected as blank is experimentally studied. The asphalt binder is modified with a virgin conventional polymer, like Styrene Butadiene Styrene, known as SBS, that has a much higher cost even considering the process benefits of recycling latex from tires. It is concluded that the changes caused mainly by the microtexture is one of the most favourable aspects for the braking distance reduction.

Keywords: recycled tires, recycling, non-skid, friction, microsurfacing 


\section{Introducción}

Se define como microaglomerado discontinuo en caliente a aquella mezcla de granulometría discontinua que es elaborada y colocada en caliente como capa de rodadura de calzadas. Sus materiales componentes son la combinación de un cemento asfáltico modificado con polímeros, áridos que presentan una discontinuidad granulométrica muy acentuada en los tamaños intermedios del total de la gradación, relleno mineral y eventualmente aditivos. Realizada la mezcla de estos materiales todas las partículas deben quedar recubiertas por una película homogénea de asfalto (CPAA, 2006). Su finalidad es dotar a la capa de rodamiento de adecuadas condiciones de resistencia mecánica, resistencia al deslizamiento y propiedades fono absorbentes.

Estas mezclas de espesor máximo de $3 \mathrm{~cm}$ utilizan por lo general como árido grueso una piedra artificial triturada de tamaño máximo $12 \mathrm{~mm}$ y un árido fino de tamaño máximo $3 \mathrm{~mm}$, presentándose como ausente la fracción entre los 3 y $6 \mathrm{~mm}$, situación que genera la discontinuidad en la curva de la mezcla de agregados. Se deben considerar dos aspectos fundamentales en el diseño y proyecto de una mezcla asfáltica (Botasso et al., 2002): a) la función resistente, que determina los materiales y los espesores de las capas a emplear en la construcción y b) la función superficial, que determina las condiciones de textura y acabado que se deben exigir a las capas superiores del camino para que resulten seguras y confortables. En el caso de los microaglomerados, éstos aportan sólo una función superficial, existiendo la necesidad de que los mismos se coloquen sobre mezclas asfálticas de mayor espesor que aporten el coeficiente estructural necesario.

\section{Fenómenos de superficie en la calzada de un pavimento}

Desde las tecnologías de las mezclas asfálticas se pueden generar condiciones superficiales que permitan que la textura de la capa de rodamiento en conjunto con las características de los neumáticos provoquen la mejor combinación posible a fin de evacuar rápidamente el agua del nivel superior del pavimento y generar la mayor adherencia neumático calzada disminuyendo el fenómeno de hidroplaneo. Para el lector interesado en mecanismos de adherencia neumático calzada se sugiere consultar por ejemplo, Dixon (1991), Gillespie (1992) y Pacejka (2002).

Las mezclas que mejor desempeño desarrollan a nivel de superficie generando texturas ásperas y rugosas son mezclas que en general serán de espesor menor a $3 \mathrm{~cm}$ denominadas microaglomerados asfálticos. Las texturas deseables se denominan (Botasso et al., 2002):

- Macrotextura: dada por la longitud de onda del tamaño del agregado grueso, es la que permite la evacuación del agua (ver Figura 1).

- Microtextura: dada por la aspereza del mastic asfáltico conformado por el ligante y los finos y fillers de la mezcla, permitiendo las mejoras en la adherencia neumático calzada.

Ambas en niveles adecuados permiten disminuir las distancias de frenado, con pavimento mojado, ya que se evacúa más rápidamente el agua y el agarre del neumático aumenta.

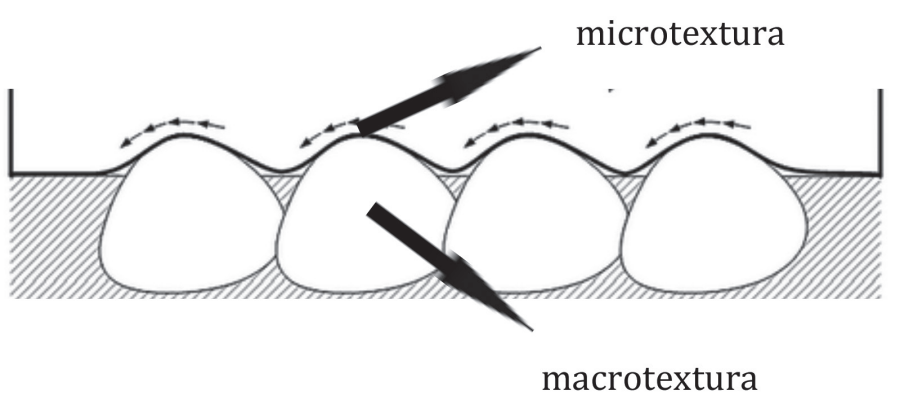

Figura 1: Macrotextura y microtextura

\section{Las dispersiones de los polímeros en el asfalto}

Para que estas mezclas denominadas antiderrapantes mantengan sus valores en el tiempo frente al amasado del tránsito y de la temperatura de la calzada, se hace necesario contar con un asfalto modificado con polímero. Los polímeros se dispersan en el asfalto aportando una fuerte energía de corte, temperatura y contando con la suficiente cantidad de hidrocarburos naftenos aromáticos y polares aromáticos que humecten al polímero y permitan que éste se hinche dentro de la masa de asfalto. Se cuentan con dos tipos de polímeros. Por un lado el polvo proveniente de la trituración ambiental de los Neumáticos Fuera de Uso NFU (Foto 1a) y por otro lado el estireno butadieno estireno SBS, polímero virgen en forma de pelets (Foto $1 b$ ). 

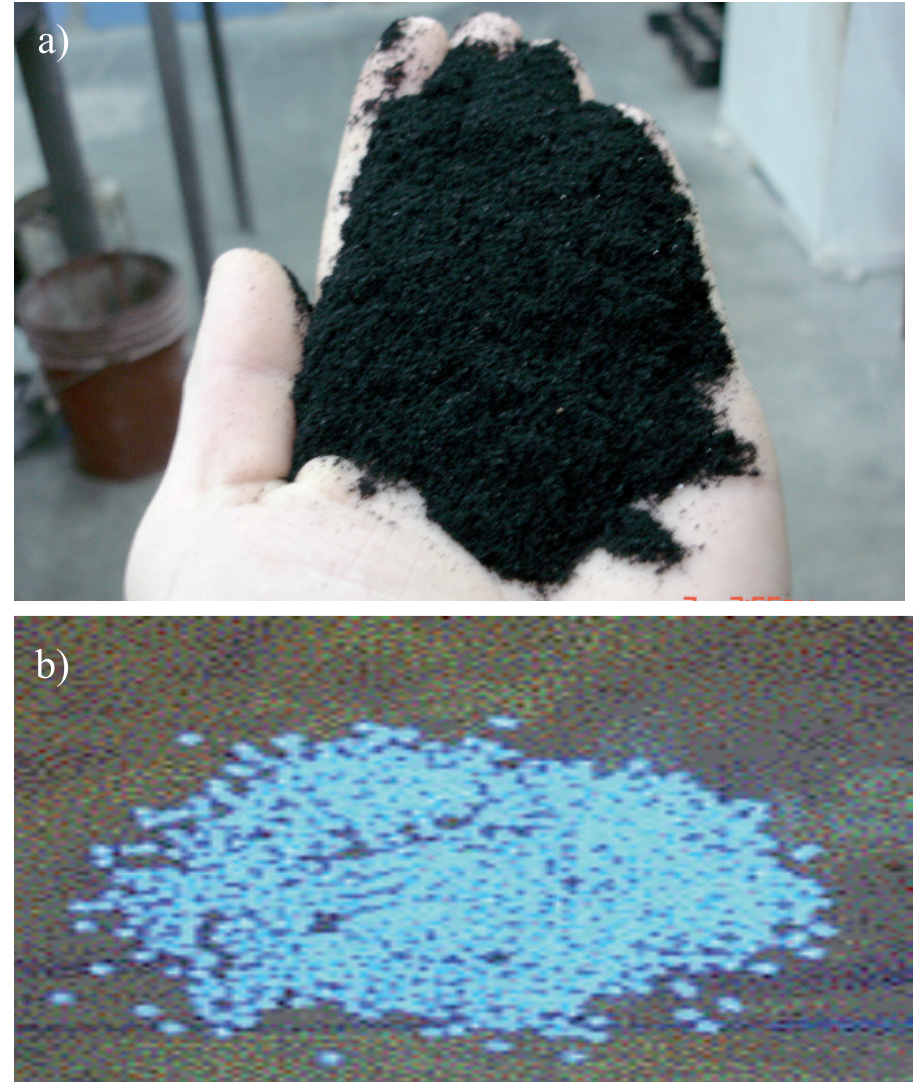

Foto 1: a) Caucho molido NFU y b) polímero SBS

El dispersor de NFU y SBS utilizado en este proceso se muestra en la Foto 2.

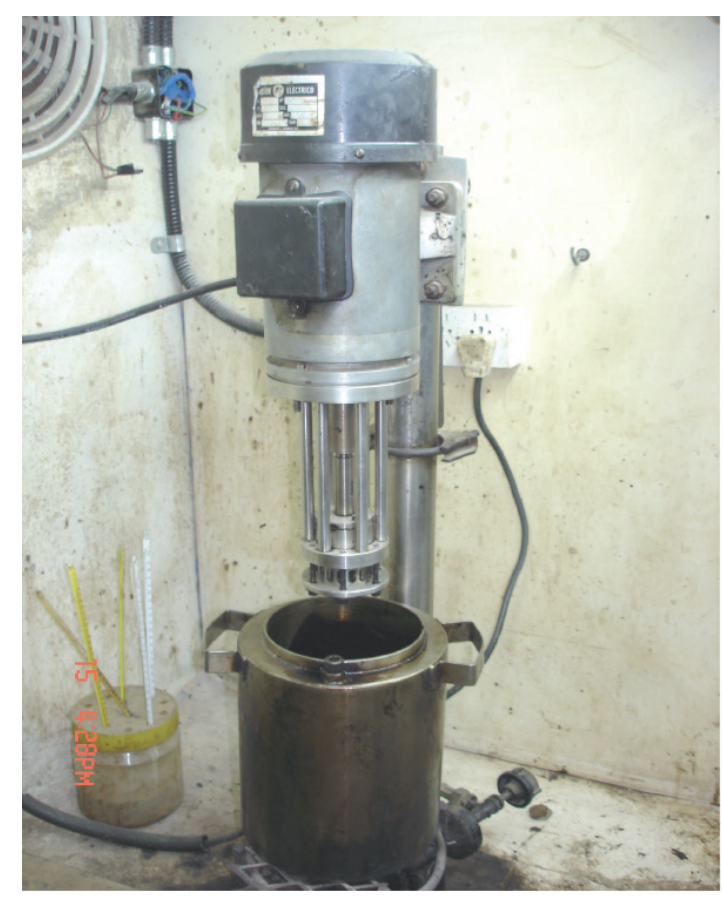

Foto 2: Equipo dispersor de NFU y SBS en asfalto
Se caracterizaron las dispersiones poliméricas en base a lo fijado por la norma IRAM 6596 (2010), en la Tabla 1 se pueden observar los valores obtenidos.

Tabla 1: Caracterización de los asfaltos modificados con NFU y con SBS

\begin{tabular}{|l|c|c|c|}
\hline Ensayo & Unidad & $\begin{array}{c}\text { CA-20+ } \\
8 \% \text { NFU }\end{array}$ & $\begin{array}{c}\text { CA-20+ } \\
3 \% \text { SBS }\end{array}$ \\
\hline Penetración & $1 / 10 \mathrm{~mm}$ & 44 & 69 \\
\hline $\begin{array}{l}\text { Punto de } \\
\text { ablandamiento }\end{array}$ & ${ }^{\circ} \mathrm{C}$ & 56 & 65 \\
\hline $\begin{array}{l}\text { Recuperación elástica } \\
\text { torsional }\end{array}$ & $\%$ & 33 & 71 \\
\hline Ductilidad a $5^{\circ} \mathrm{C}$ & $\mathrm{cm}$ & 15 & 42 \\
\hline
\end{tabular}

\section{Características de la mezcla}

Las mezclas asfálticas de superficie trascienden el diseño de laboratorio convencional. Vale decir no alcanza valores de estabilidad, flujo o vacíos, sino que se necesita considerar la prestación en obra una vez colocada a fin de valorar su desempeño en condición de pavimento mojado frente a un frenado de emergencia, situación considerada crítica en la distancia que recorre un vehículo al intentar esa maniobra.

En general se clasifican como:

- MAC M8 y M10: microconcretos asfálticos en caliente con granulometría discontinua, monogranulares de tamaño máximo de agregados 8 y $10 \mathrm{~mm}$ respectivamente, especialmente proyectados para capas de entre 15 y $25 \mathrm{~mm}$.

- MAC F8 y F10: microconcretos asfálticos en caliente con granulometría discontinua de tamaño máximo de agregados 8 y $10 \mathrm{~mm}$ respectivamente, especialmente proyectados para capas de entre 20 y $25 \mathrm{~mm}$ los MAC F8 y entre 25 y $35 \mathrm{~mm}$ los MAC F10.

En el presente trabajo se diseña un MAC F10 con asfalto modificado con NFU y SBS. La macrotextura se puede medir en forma puntual con un ensayo denominado parche de arena según la norma IRAM 1555 (2010), ver Foto 3. La microtextura se puede medir en forma puntual con un ensayo denominado péndulo inglés TRRL según la norma IRAM 1850 (2010), ver Foto 4. 


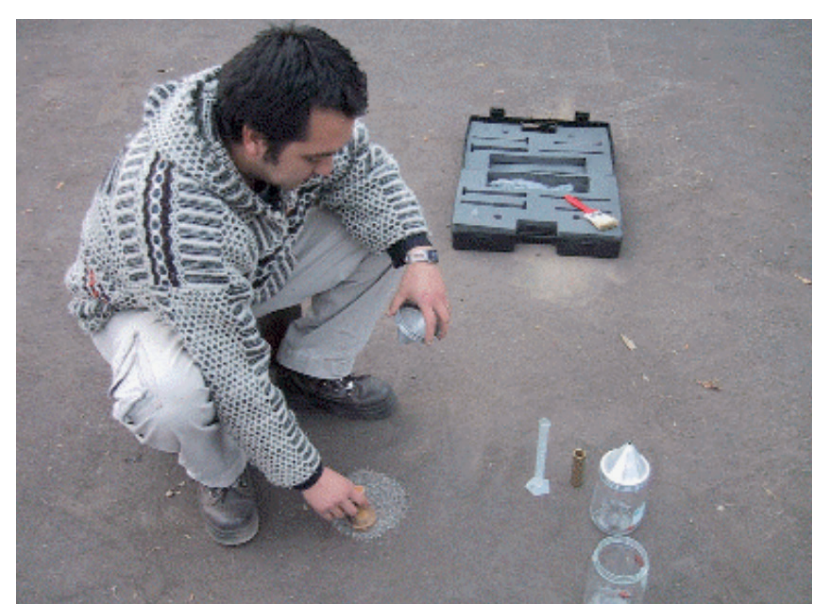

Foto 3: Parche de arena

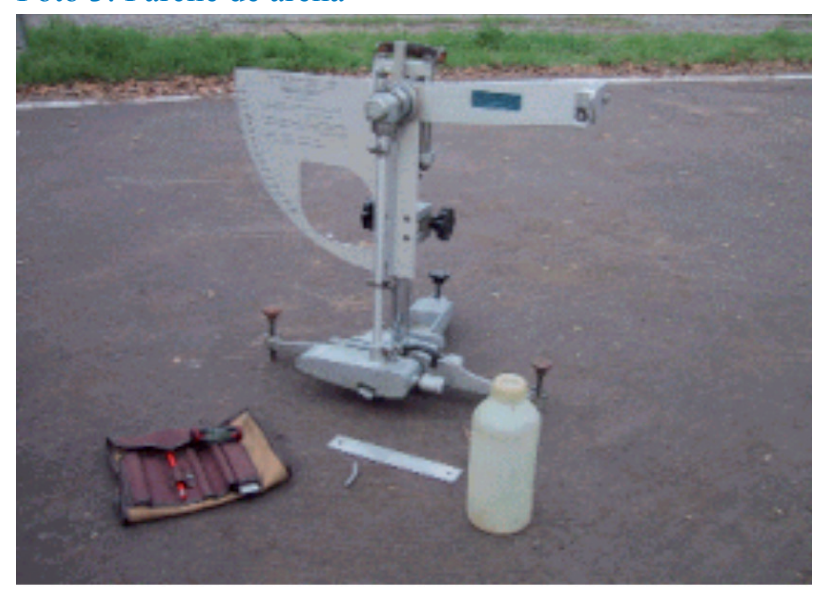

Foto 4: Péndulo TRRL

Los valores exigidos habitualmente en las especificaciones se presentan en la Tabla 2.

Tabla 2: Valores exigibles de macro y micro textura

\begin{tabular}{|l|c|c|}
\hline Tipo de textura & Norma & F8 - F10 \\
\hline $\begin{array}{l}\text { Macrotextura, altura } \\
\text { de círculo de parche de } \\
\text { arena, mm }\end{array}$ & $\begin{array}{c}\text { IRAM 1850 } \\
(2010)\end{array}$ & $\begin{array}{c}\text { Promedio del lote } \\
>1.1 \\
\text { Mímo absoluto } \\
>0.8\end{array}$ \\
\hline $\begin{array}{l}\text { Microtextura, coeficiente } \\
\text { de resistencia al } \\
\text { ahuellamiento, BPN }\end{array}$ & $\begin{array}{c}\text { NLT 175 } \\
\text { (2005) }\end{array}$ & Mínimo 0.65 \\
\hline
\end{tabular}

\section{Diseño del modelo de laboratorio}

No se ha desarrollado aún en laboratorio, según han indagado los autores en la literatura técnica, un modelo que permita predecir la futura macro y microtextura de una mezcla, antes de ser colocada al menos en un tramo de prueba. Menos aún evaluar resultados instantáneos para ciclos de temperatura y tránsito, observando el deterioro de la macro y microtextura de una mezcla con aumentos en la aplicación del número de pasadas de ejes del tránsito.

En el presente trabajo se ha desarrollado un experimento que utiliza modernos ensayos dinámicos diseñados para otros fines y que dadas sus características pueden constituirse en el modelo buscado. Se propone que en laboratorio se puedan hacer modelos que superen la escala de una probeta del ensayo Marshall, constituyendo una pieza de mayor superficie, de $30 \mathrm{~cm}$ x $30 \mathrm{~cm}$, en donde las condiciones de texturas puedan ser observadas, como se muestra en la Foto 5 y en la Figura 2.
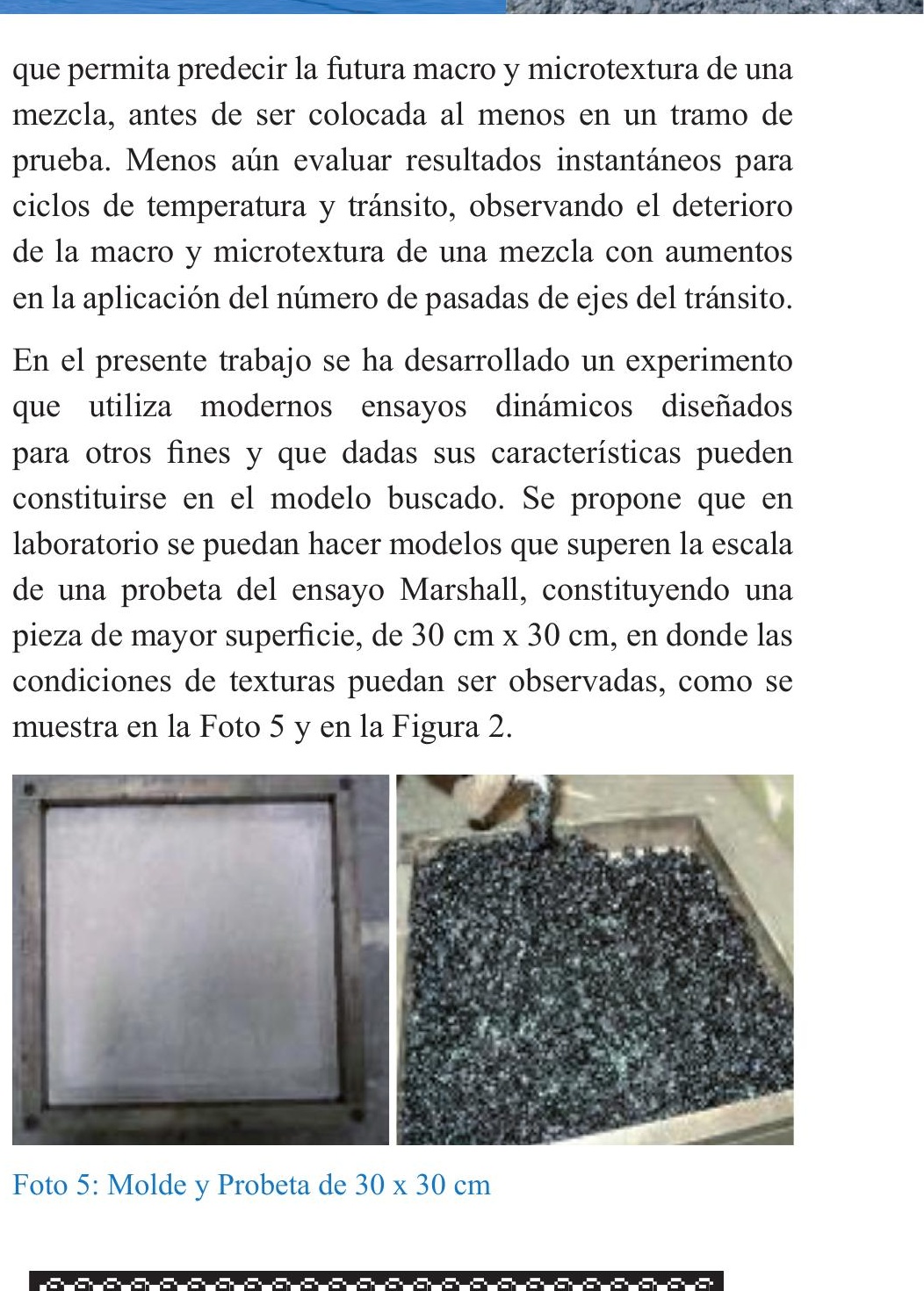

Foto 5: Molde y Probeta de 30 x $30 \mathrm{~cm}$

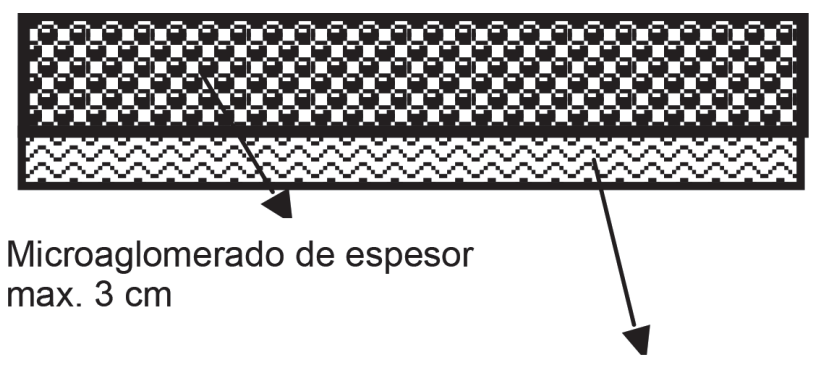

Base rígida de espesor de $2 \mathrm{~cm}$

Figura 2: Esquema del modelo de ensayo

El modelo de probeta proviene del ensayo de ahuellamiento (wheel tracking test) estandarizado en la norma BS EN 12697-22 (2003) y homologada en Argentina para estudiar las deformaciones plásticas permanentes en las mezclas asfálticas de capacidad estructural, por acción del tránsito pesado. El equipo mostrado en la Foto 6 aplica en la probeta una carga con ciclos sinusoidales, tal cual lo hace el tránsito en la calzada con una frecuencia de 26.5 ciclos por 
minuto, pudiendo variarse la temperatura de ensayo según las condiciones climáticas de diseño en donde se encuentre la obra. En el caso de ahuellamiento este ensayo se realiza a $60^{\circ} \mathrm{C}$, pues es donde se pone de manifiesto este tipo de fenómeno. La idea es que esta carga sea la que produzca el deterioro de las condiciones de superficie para los dos tipos de modificadores utilizados en el microaglomerado.

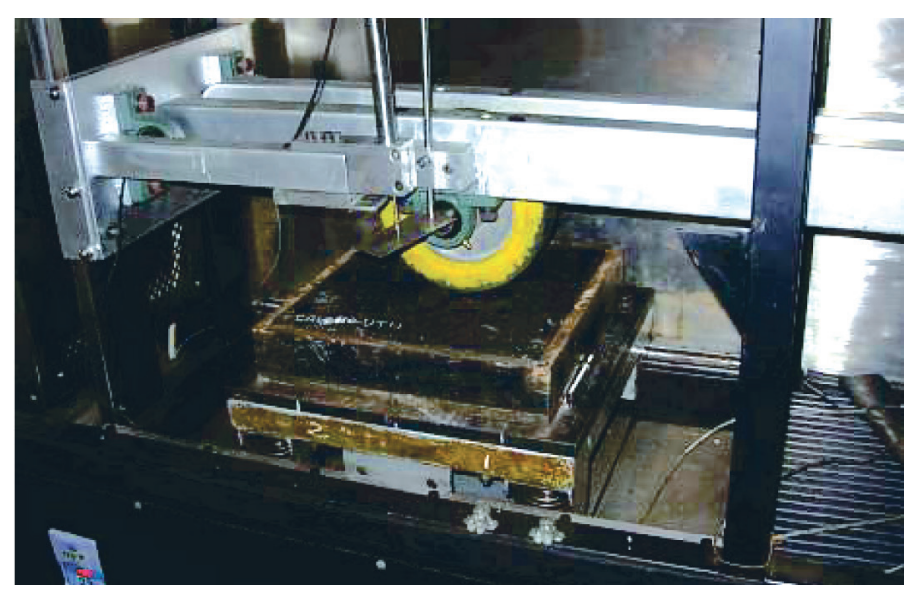

Foto 6: Equipo de wheel tracking test

La Foto 7 muestra el moldeo de una probeta con un equipo de compactación que simula las condiciones mínimas de compactación que una micromezcla necesita en la obra.

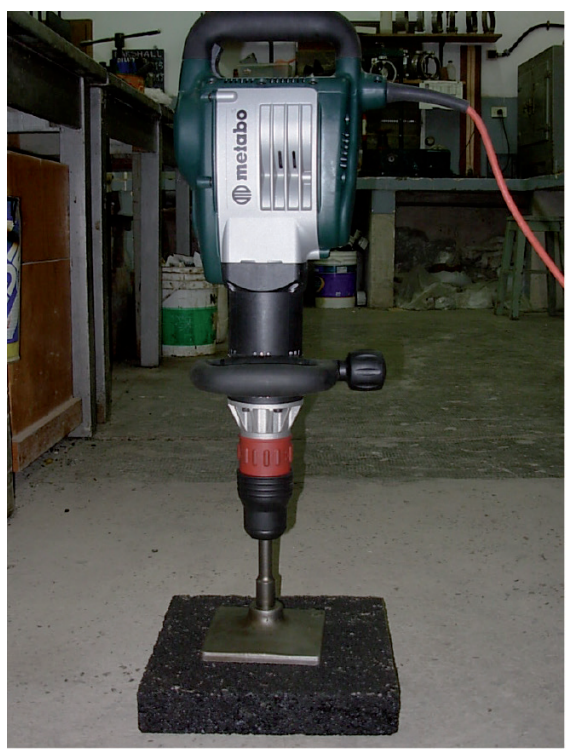

Foto 7: Equipo de compactación de la muestra

Se pretende de esta forma conformar las mezclas tipo microaglomerado discontinuo en caliente utilizando los dos tipos de modificadores del ligante asfáltico, el SBS y el
NFU. Una vez obtenidas estas muestras se procede sobre esta superficie a medir la macrotextura en forma puntual con el ensayo del parche de arena y la microtextura en forma puntual con el péndulo inglés TRRL en condición de pavimento mojado. Luego de esta determinación se somete a las muestras a la acción de la rueda del equipo de wheel tracking test, con el fin de que simule la acción del tránsito y provoque el deterioro en las condiciones iniciales de textura. La mezcla utilizada es una MAC F10 con las características presentadas en la Tabla 3.

Tabla 3: Dosificación de las mezclas diseñadas con asfalto modificado con NFU y SBS

\begin{tabular}{|l|c|c|}
\hline $\begin{array}{l}\text { Materiales } \\
\text { componentes }\end{array}$ & Nomenclatura & $\begin{array}{c}\text { Porcentajes en } \\
\text { mezcla }\end{array}$ \\
\hline $\begin{array}{l}\text { Piedra triturada } \\
\text { granítica }\end{array}$ & $6: 12$ & 62.4 \\
\hline $\begin{array}{l}\text { Arena granítica de } \\
\text { trituración }\end{array}$ & $0: 3$ & 27.4 \\
\hline Filler: Cal & cal & 4.8 \\
\hline \multirow{2}{*}{$\begin{array}{l}\text { Asfalto utilizados en } \\
\text { cada caso }\end{array}$} & CA-20+8\% de NFU & 5.4 \\
\cline { 2 - 3 } & CA-20+3\% SBS & 5.4 \\
\hline
\end{tabular}

Para el diseño de la mezcla se ha utilizado agregado granítico y un cemento asfáltico que por sus valores de viscosidad clasifica como un CA-20. La cantidad de asfalto en cada mezcla fue el mismo, un 5,4\% en peso de la mezcla. La cantidad de ligante asfáltico se ha determinado como el valor óptimo de las mezclas. La cantidad óptima que permitió alcanzar valores similares de desempeño entre los dos ligantes fueron la adición del $8 \%$ de NFU y del $3 \%$ de SBS. La Foto 8 muestra el proceso de formación.

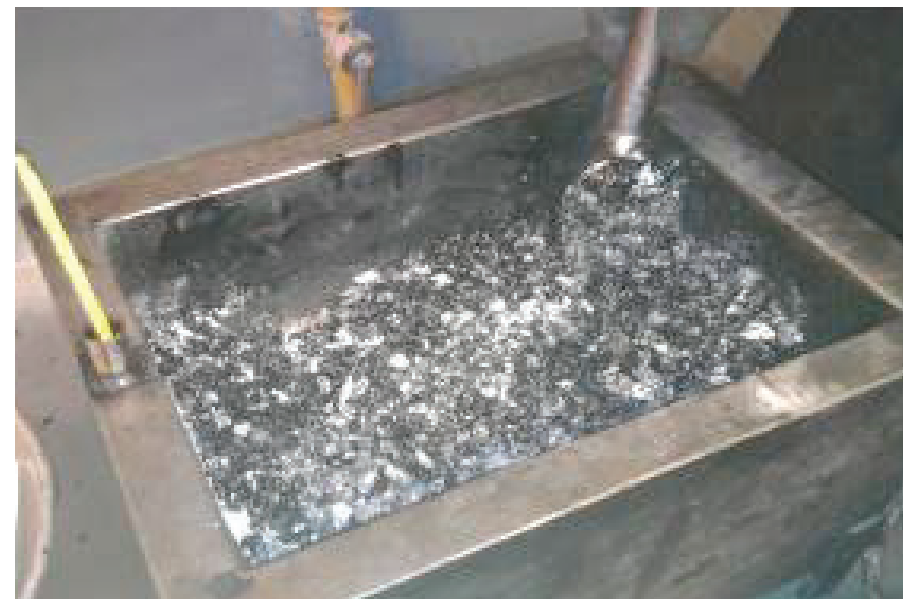

Foto 8: Elaboración de la mezcla 
En la Tabla 4 se presentan los datos que permiten construir las curvas granulométricas mostradas en el gráfico de la Figura 3, donde se puede observar la curva de la mezcla MAC F10 discontinua entre los tamices ASTM No 4 y 8.

Tabla 4: Granulometría de la mezcla

\begin{tabular}{|c|c|c|c|c|}
\cline { 3 - 5 } \multicolumn{2}{c|}{} & \multicolumn{3}{c|}{$\%$ que pasa } \\
\hline Tamiz & abert., $\mu \mathrm{m}$ & c. $\min$ & mezcla & c. $\max$ \\
\hline $1 / 2$ & 12700 & 100 & 100 & 100 \\
\hline $3 / 8$ & 9500 & 75 & 94.60 & 97 \\
\hline $1 / 4$ & 6250 & 40 & 58.68 & 65 \\
\hline 4 & 4750 & 25 & 32.93 & 40 \\
\hline 8 & 2360 & 20 & 25.74 & 35 \\
\hline 30 & 600 & 12 & 12.96 & 25 \\
\hline 200 & 75 & 7 & 7.88 & 10 \\
\hline
\end{tabular}

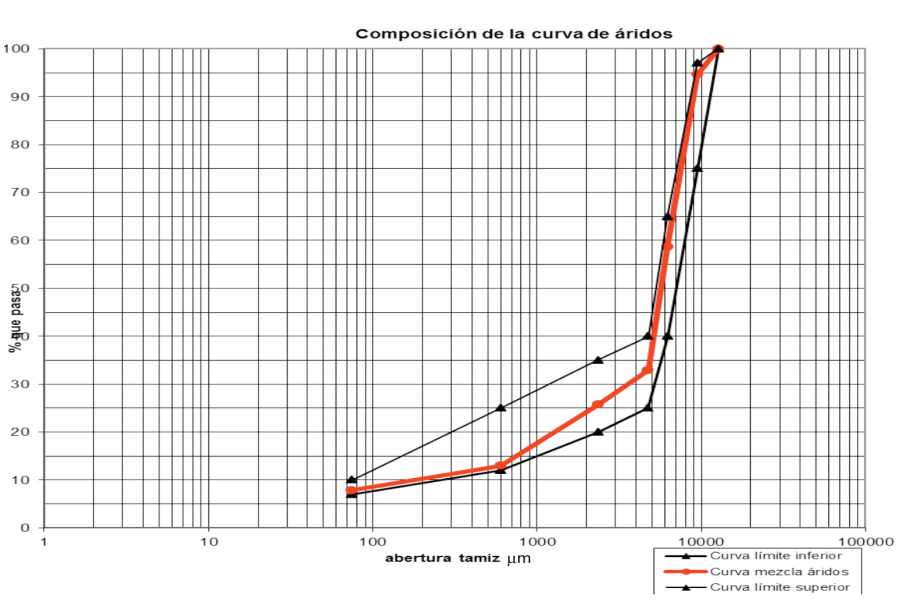

Figura 3: Curva de la mezcla y límites

Luego se procede al diseño con la metodología Marshall, la cual arrojó los valores volumétricos y mecánicos mostrados en la Tabla 5.

Tabla 5: Valores volumétricos y mecánicos del ensayo Marshall

\begin{tabular}{|c|c|c|c|c|c|c|c|}
\hline $\begin{array}{c}\text { Mezcla } \\
\text { asfáltica }\end{array}$ & $\begin{array}{c}\text { Densidad } \\
\text { Marshall, } \\
\mathrm{g} / \mathrm{cm}^{3}\end{array}$ & $\begin{array}{c}\text { Densidad } \\
\text { Rice, } \\
\mathrm{g} / \mathrm{cm}^{3}\end{array}$ & $\begin{array}{c}\text { Vacíos, } \\
\%\end{array}$ & $\begin{array}{c}\text { Estab., } \\
\mathrm{kg}\end{array}$ & $\begin{array}{c}\text { Fluencia, } \\
\mathrm{mm}\end{array}$ & $\begin{array}{c}\text { Vacíos } \\
\text { agreg. } \\
\text { mineral, } \\
\%\end{array}$ & $\begin{array}{c}\text { Relac. } \\
\text { betún } \\
\text { vacíos, } \\
\%\end{array}$ \\
\hline $\begin{array}{c}\text { Modificado } \\
\text { con 8\% de } \\
\text { caucho }\end{array}$ & 2.302 & 2.453 & 6.2 & 875 & 4.3 & 18.6 & 66.8 \\
\hline $\begin{array}{c}\text { Modificado } \\
\text { con SBS }\end{array}$ & 2.312 & 2.443 & 5.4 & 1096 & 4.4 & 17.9 & 69.7 \\
\hline
\end{tabular}

Los valores obtenidos tanto en forma volumétrica como mecánica cumplen con lo exigido para este tipo de mezclas discontinuas en las especificaciones técnicas vigentes en Argentina para mezclas de bajo espesor redactadas por la
CPAA (2006). Luego se procede al moldeo de las probetas para el modelo seleccionado dando como resultado las muestras que se presentan en la Foto 9.
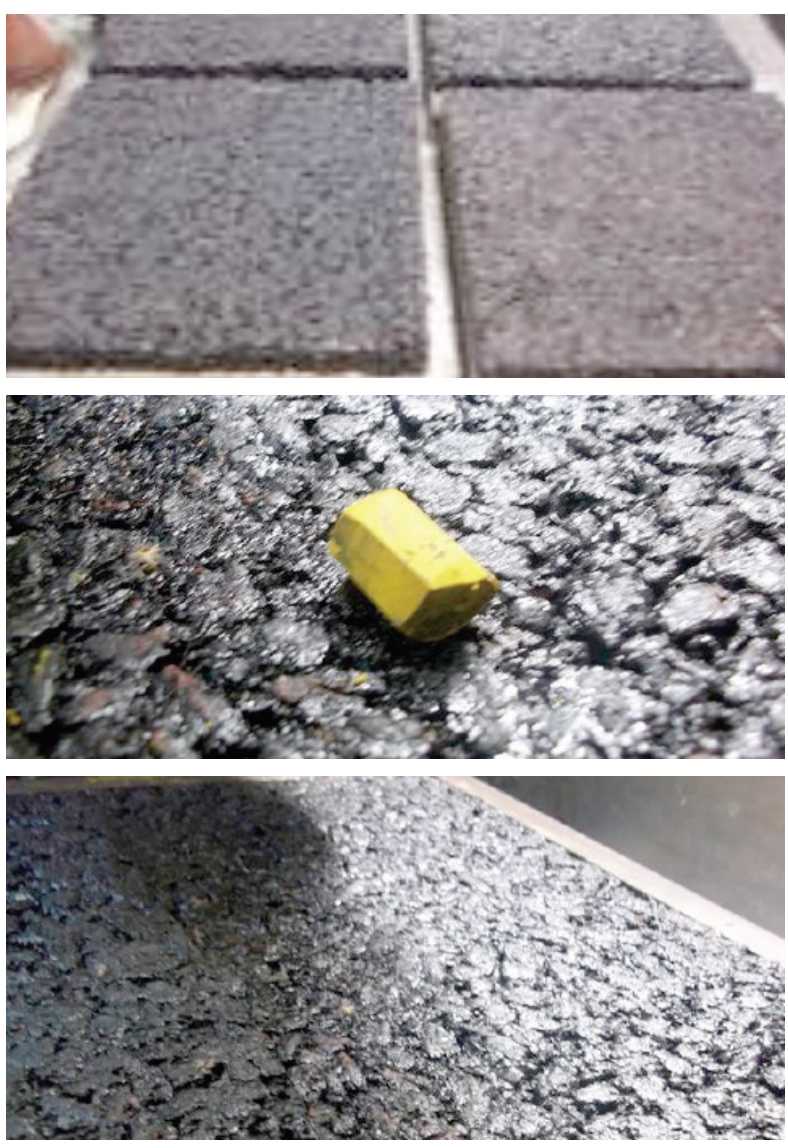

Foto 9: Muestras de 30 x 30 de MAC F10 con asfaltos con NFU y SBS

Una vez confeccionadas las probetas de 30 x $30 \mathrm{~cm} \mathrm{y}$ acondicionadas durante dos horas a $60^{\circ} \mathrm{C}$, se procede a la utilización del equipo de wheel tracking test WTT para la simulación del tránsito sobre las probetas. Dicha simulación se realizará en tres franjas contiguas (cada una de ancho igual a la de la rueda) sobre la superficie de las probetas, esto con el fin de generar una superficie lo suficientemente ancha que permita la realización de los ensayos de evaluación de macro y microtextura luego de la simulación del tránsito. En la Foto 10 se muestran imágenes del proceso de ensayo cíclico.

En todos los casos se ha moldeado un total de 10 probetas para cada condición de ensayo. La solicitación generada por la rueda del equipo WTT, resulta ser un factor de deterioro de la macro y microtextura. 

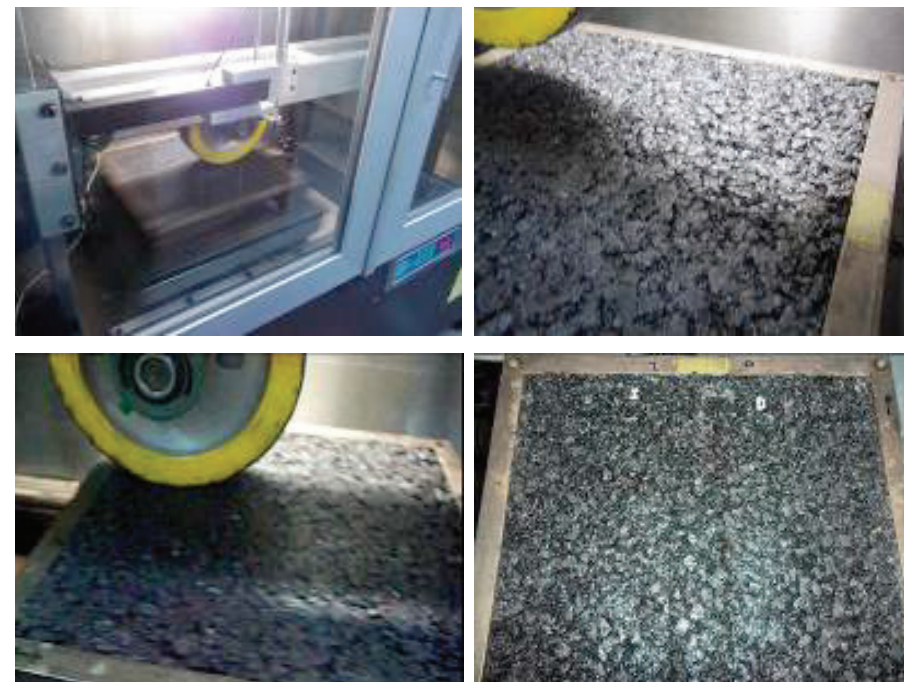

Foto 10: Procedimiento de carga con el WTT en las probetas - 3 pasadas una al lado de la otra

\section{Macrotextura antes y después del tránsito con el equipo WTT}

Las determinaciones de macro y microtextura se realizan con el equipo de parche de arena y con el péndulo inglés respectivamente. Las medidas puntuales de macrotextura consisten en extender sobre la superficie de un pavimento un volumen determinado de arena fina uniforme que se distribuye y enrasa mediante un esparcidor, quedando la arena enrasada con las puntas más salientes. Se procura extender la arena formando un círculo, con lo que es fácil determinar el área cubierta por la arena. A partir del volumen de arena utilizado y del área de pavimento cubierta por ella, se calcula una profundidad media de los huecos rellenos por la arena, valor que se utiliza como medida de la macrotextura superficial del pavimento (ver Foto 11). En la Tabla 6 se pueden observar los valores medios de macrotextura en los dos microaglomerados y la disminución de este parámetro cuando fue sometido a la acción del tránsito durante 2 horas en cada franja, totalizando un total de tres franjas de carga a una temperatura extrema de $60^{\circ} \mathrm{C}$.

Tabla 6: Deterioro de la macrotextura

\begin{tabular}{|l|c|c|c|}
\hline $\begin{array}{l}\text { Mezcla } \\
\text { MAC F10 }\end{array}$ & Antes WTT & Después WTT & $\begin{array}{c}\text { Disminución } \\
\text { macrotextura, \% }\end{array}$ \\
\cline { 2 - 3 } Con NFU & 1.100 & 0.591 & 46.3 \\
\hline Con SBS & 1.114 & 0.684 & 38.6 \\
\hline
\end{tabular}
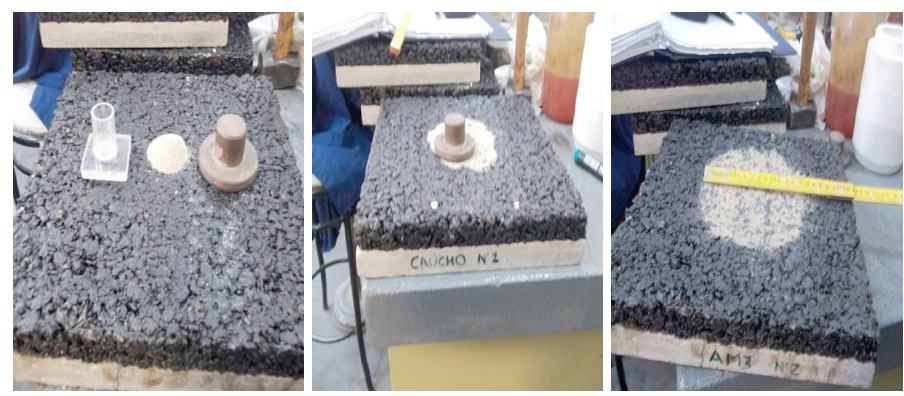

Foto 11: Medición de la macrotextura con el parche de arena sobre el modelo

\section{Microtextura antes y después del tránsito con el equipo WTT}

La microtextura se puede medir en forma puntual con el ensayo denominado péndulo inglés TRRL (IRAM 1850). Este ensayo consiste en medir la pérdida de energía de un péndulo de características conocidas, provisto en su extremo de una zapata de caucho, cuando la arista de la zapata roza sobre la superficie a ensayar, con una presión determinada, a lo largo de una longitud fija. Esta pérdida de energía se mide por el ángulo suplementario de la oscilación del péndulo. Esta operación se realizó antes y después de la simulación del tránsito en el modelo. Los resultados se presentan en la Tabla 7 y mediciones con el péndulo TRRL se muestra en la Foto 12.

Tabla 7: Deterioro de la microtextura

\begin{tabular}{|c|c|c|c|}
\hline \multirow[b]{2}{*}{$\begin{array}{l}\text { Mezcla } \\
\text { MAC F10 }\end{array}$} & $\begin{array}{l}\text { Antes } \\
\text { WTT }\end{array}$ & $\begin{array}{c}\text { Después } \\
\text { WTT }\end{array}$ & \multirow{2}{*}{$\begin{array}{c}\text { Disminución } \\
\text { microtextura, } \\
\%\end{array}$} \\
\hline & $\begin{array}{l}\text { Coef. } \\
\text { BPN }\end{array}$ & $\begin{array}{l}\text { Coef. } \\
\text { BPN }\end{array}$ & \\
\hline Con NFU & 0.882 & 0.838 & 5.0 \\
\hline Con SBS & 0.773 & 0.750 & 3.0 \\
\hline
\end{tabular}
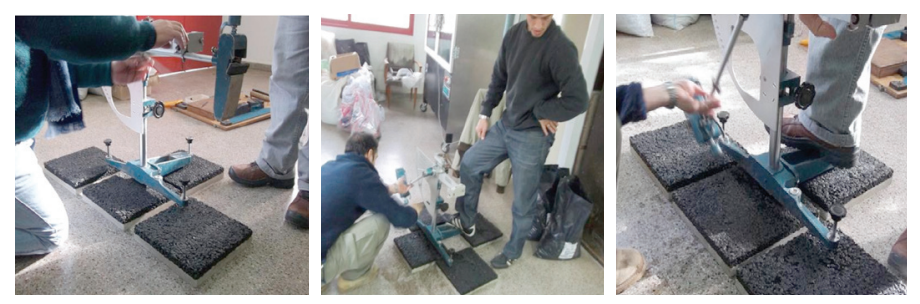

Foto 12: Medición de la Microtextura con péndulo TRRL sobre el modelo 


\section{Análisis de los resultados obtenidos}

A la luz de los experimentos realizados se puede observar en el proceso de caracterización desarrollado las siguientes particularidades. Al dispersar polvo de NFU en el cemento asfáltico CA-20 utilizado se ha logrado aumentar el punto de ablandamiento del ligante asfáltico original y aumentar la recuperación elástica por torsión. Para ello el equipo dispersor ha demostrado ser una herramienta que permite la microdispersión.

Se observa que la densidad Marshall alcanzada con el asfalto modificado con $8 \%$ de NFU es menor que la que se obtiene con el asfalto modificado con SBS. Esto posiblemente se deba al efecto de amortiguación que genera el polvo de caucho triturado en la mezcla asfáltica. De hecho el $8 \%$ fue el porcentaje máximo admitido entre los valores de modificación óptimos del ligante asfáltico y las pérdidas de densidad en las mezclas, siendo estas dos valoraciones, grado de modificación alcanzado por el asfalto versus densidad de las mezclas, dos valores que se tensionan entre sí. Los valores obtenidos volumétricos y mecánicos cumplen con lo exigido en las especificaciones para este tipo de mezclas discontinuas finas.

Las mediciones en los modelos realizados han permitido evaluar la macrotextura y la microtextura. Los valores de macrotextura son un poco menor en el microaglomerado con caucho que en el realizado con polímero virgen. Esto posiblemente se deba a la mayor cantidad de vacíos obtenidos en la mezcla con adición de NFU. La pérdida de macrotextura en la mezcla realizada con asfalto modificado con caucho de NFU, es mayor que en el microaglomerado convencional; también se puede inferir que esto sea debido al mayor porcentaje de vacíos de la mezcla. Sin embargo, ambas pérdidas mantienen entornos razonables de serviciabilidad dadas las condiciones de temperatura de $60^{\circ} \mathrm{C}$ a las que se han sometido en el modelo de ensayo WTT. En ambos casos resultan ser superiores a los valores habitualmente exigidos para planificar una próxima intervención superficial debido al deterioro de la macrotextura, este umbral es de $\mathrm{H}=0.5 \mathrm{~mm}$.

Los valores de microtextura obtenidos antes y después del proceso de solicitación del modelo son satisfactorios y los microaglomerados desarrollados con polvo de NFU tienen mejor desempeño. Esto se presume se deba a que el mastic asfáltico generado en la envuelta del árido grueso, que constituye la microtextura, es más áspero debido a la presencia de las partículas microdispersas del polvo de NFU en el mezcla.

Finalmente, se debe correlacionar los resultados obtenidos en laboratorio con los obtenidos en una obra a escala real para condiciones iniciales y después del deterioro, considerando condiciones climáticas y de tránsito particulares del proyecto en estudio.

\section{Conclusiones}

Se concluye que es posible aplicar el modelo de solicitación del ensayo de Wheel Tracking Test WTT como modelo de carga y solicitación para el deterioro de la macrotextura y de la microtextura de un microaglomerado discontinuo en caliente. Se evidencian cambios de significación en estas propiedades, que si bien no se pueden asociar al ciclo real de deterioro de la calzada, sí permite establecer correlaciones comparativas entre diferentes mezclas hasta tanto se establezcan relaciones con los modelos a escala real en la calzada de la obra.

El asfalto modificado con caucho proveniente de la trituración de neumáticos fuera de uso NFU permite desarrollar microaglomerados con valores de macrotextura y microtextura iniciales similares a los de una mezcla realizada con polímero virgen. Luego de poner las probetas de $30 \times 30 \mathrm{~cm}$ en el equipo WTT, se observó que al someterlas a un proceso de 3 pasadas, a efectos de generar un área de valoración adecuada, las dos variables evaluadas se encuentran dentro de un rango de apreciación esperable. La macrotextura de las mezclas con NFU se deterioran más rápidamente que en un microaglomerado con polímero virgen, influyendo esto en la capacidad de evacuación del agua de la superficie de la calzada. Sin embargo, los valores en ambos casos, antes y después del proceso de deterioro, son aún satisfactorios. En cuanto a la microtextura, la responsable de disminuir la distancia de frenado en condición de pavimento mojado, se ha visualizado un mejor desempeño en las mezclas realizadas con asfalto modificado con NFU. Esto se presume se debe a las condiciones de aspereza que el mastic adquiere con el polvo de neumáticos depositado sobre los áridos gruesos de la misma. 
Se plantea como futuro trabajo de investigación, correlacionar los valores alcanzados en laboratorio, tanto para las condiciones iniciales, como después del deterioro, con valores de deterioro percibidos en las obras, con condiciones climáticas y de tránsito de la región donde se realice la obra.

\section{Referencias}

Botasso G., González, R. y Rivera J. (2002). Nuevas Mezclas Asfálticas. LEMaC Centro Investigaciones Viales UTN Reg. La Plata, Argentina

BS EN 12697-22 (2003). Bituminous mixtures. Test methods for hot mix asphalt. Wheel tracking. British Standard Institution, London

CPAA Comisión Permanente del Asfalto de Argentina (2006). Especificaciones Técnicas de mezclas asfálticas en caliente de bajo espesor. Versión 01. Buenos Aires, Argentina

Dixon, J.C. (1991). Tyres, suspension and handling. Cambridge University Press
Gillespie, T.D. (1992). Fundamentals of Vehicle Dynamics. SAE Inc.

IRAM 1555 (2010). Agregados. Determinación del coeficiente de resistencia al deslizamiento con el péndulo TRRL. Instituto de Normalización Argentino. Buenos Aires, Argentina

IRAM 6596 (2010). Asfaltos modificados con polímeros para uso vial. Clasificación y requisitos. Instituto de Normalización Argentino. Buenos Aires, Argentina

IRAM 1850 (2010). Agregados. Método de determinación de la profundidad de la macrotextura superficial de un pavimento mediante el círculo de arena. Instituto de Normalización Argentino. Buenos Aires, Argentina

NLT 175 (2005). Coeficiente de resistencia al deslizamiento (CRD) con el péndulo del TRRL. Normas del Ministerio de Fomento de España.

Pacejka, H. B. (2002). Tyre and Vehicle Dynamics. Butterworth Heinemann 